\title{
Replenishing the damaged heart with oxygen by nature-inspired photosynthesis
}

\author{
Juan Tang ${ }^{1}$, Kathy O Lui ${ }^{2} \&$ Bin Zhou*,1 \\ ${ }^{1}$ The State Key Laboratory of Cell Biology, Chinese Academy of Sciences, Shanghai, 200031, PR China \\ ${ }^{2}$ Department of Chemical Pathology, Li Ka Shing Institute of Health Sciences, The Chinese University of Hong Kong, 999077, PR \\ China \\ * Author for correspondence: zhoubin@sibs.ac.cn
}
"Intriguingly, Joseph Woo and colleagues have recently reported that the photosynthesis process that happens in trees can be applied in the injured myocardium for oxygen supply [5]."

First draft submitted: 12 September 2017; Accepted for publication: 9 November 2017; Published

online: 16 January 2018

Keywords: cardiology • heart • oxygen • regeneration $\bullet$ repair

Acute myocardial infarction (MI), commonly known as heart attack, remains the leading cause of morbidity and mortality worldwide [1]. Owing to blockage of the coronary artery by a blood clot formed after rupture of the atherosclerotic plaque, the heart muscle dies as a result of a sudden deprivation of circulating blood that supplies essential oxygen. Currently, the only way to treat coronary heart disease is to rebuild blood flow to the heart. One conventional treatment in the clinic is coronary artery bypass grafting, which creates a new path for oxygenrich blood to flow back to the heart muscle. Alternatively, a nonsurgical method, namely percutaneous coronary intervention, opens the blocked vessels through catheterization and restores local blood flow. Recent studies also suggest that stem cell transplantation could promote neovascularization after acute MI through paracrine secretion of cytokines and angiogenic factors [2]. However, a series of complications exist with these interventions, some patients are ineligible for such treatments and long-term efficacy of some conventional treatments remain controversial [3,4]. Thus, new insights into the development of alternative strategies for supplying oxygen efficiently to the heart muscle are of utmost clinical relevance.

In nature, oxygen is supplied by plants through a process called photosynthesis, which converts light energy into chemical energy. In plant cells, the chloroplast is the specialized organelle responsible for photosynthesis, where synthesis of oxygen and sugars from carbon dioxide and water takes place. Whether this naturally built system for plants could be utilized for supplying oxygen to the infarcted myocardium remains unknown and untested in any medical setting.

Intriguingly, Joseph Woo and colleagues have recently reported that the photosynthesis process that happens in trees can be applied in the injured myocardium for oxygen supply [5]. They demonstrated that a bacterium Synechococcus elongatus, like the chloroplast in plants, could also photosynthesize after light exposure. While $S$. elongatus consumes carbon dioxide and produces oxygen by photosynthesis, animal cells consume oxygen and produce carbon dioxide by respiration. Therefore, the research team placed both the bacteria and mouse cardiomyocytes together in petri dishes, so the bacteria converted carbon dioxide and water into oxygen and glucose in the light. They found that $S$. elongatus promoted a substantial increase in oxygen tension and enhanced cellular metabolism of cardiomyocytes. Next, Woo and colleagues took a bold step to test if application of S. elongatus in live animals could provide oxygen to the infarcted myocardium that was deprived of blood flow (Figure 1). By generating a rat MI model, they applied an intramyocardial injection of $S$. elongatus. In just $10 \mathrm{~min}$, the oxygen level in the illuminated and nonilluminated parts of the heart increased by nearly 25 - and three-fold, respectively. Importantly, light exposure on injected bacteria enhanced ventricular function during acute ischemia and exerted a far-reaching protective benefit in long-term ventricular performance [5]. The long-term functional benefit of such a photosynthetic therapy as a result of short-term light exposure $(\sim 1-2 \mathrm{~h})$ is surprising and amazing. This therapeutic efficacy might be due to an increased tissue oxygenation during the critical time window post-MI, 


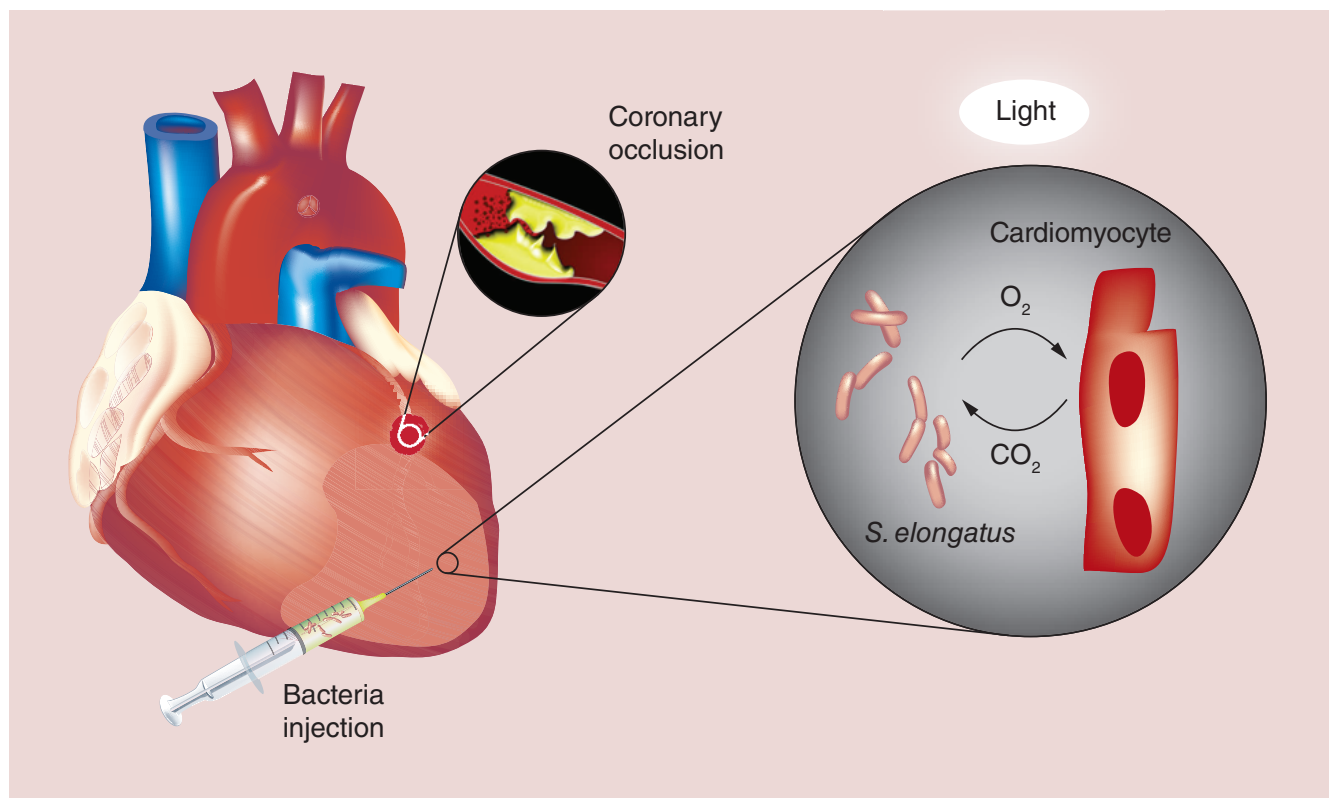

Figure 1. Photon-powered myocardium by bacteria. Left panel shows ischemia after coronary artery blockage and injection of the bacterium Synechococcus elongatus into the infarcted myocardium. Right panel shows light-induced production of oxygen by bacteria for replenishing the cardiomyocytes.

allowing a large number of cardiomyocytes to survive when blood flow partially recovered through new collateral vessel formation subsequently [6]. Since healing of acute MI involves a series of complex yet coordinated processes such as neovascularization, inflammation, cell apoptosis and necrosis [7-9], oxygen-releasing bacteria might possibly exert some beneficial effects on the recruited inflammatory cells or resident endothelial cells shortly after cardiac injury. Nevertheless, the underlying cellular and molecular mechanisms merit further investigations in the future.

The supply of a 'miniature tree' to the injured heart via a bacterial vaccine certainly represents a conceptually novel and cutting-edge advancement in the cardiovascular field. Not only does this work contribute a new therapeutic potential to cardiac injury, it also provides implications for other ischemic diseases such as stroke, pulmonary arterial hypertension and tumor angiogenesis. The oxygen-producing bacteria could also be used in the transplantation setting as transient deprivation of blood flow is confronted during organ transplantation. Thus, delivering the cyanobacteria to specific transplantation sites could preserve organ function against oxygen deprivation. Like any great study, this beautiful piece of work opens new avenues for questions and room for improvement.

For translational application of this novel strategy in the future, some technical hurdles need to be resolved. Although most bacteria were cleared from the heart tissue, more comprehensive analysis on the systemic immune response should be monitored to ensure that the bacteria are nontoxic and benign to organs of the whole body. While clearance of the majority of bacteria from the injected heart was fast (within $1 \mathrm{~h}$ ), it is also equally important to retain them for a little bit longer (from hours to a few days) to maximize their effects in the tissue before collateral coronary artery forms to maintain a sustainable regional blood flow. It would be equally important to genetically improve production of oxygen as well as survival of the bacteria. Moreover, the light source for internal organs such as the heart needs further optimization as the current method relied on a direct light exposure on bacteria, which would require open-chest operation and exposure in the air. It could be more ideal if a new type of bacteria can be engineered to utilize the far-red spectrum of light to produce oxygen, potentially facilitating activation by transcutaneous delivery of light. Furthermore, currently the bacteria only supplied oxygen but not glucose to cardiomyocytes. Since both oxygen and glucose are products of photosynthesis, the genetically engineered bacteria that export both oxygen and glucose to cardiomyocytes might offer more potent therapeutic efficacy to the injured myocardium. Last but not least, the supply of oxygen-producing bacteria could also be combined with cell or cytokine replacement therapy to maximize the therapeutic efficacy, salvaging cardiomyocytes after acute MI and mitigating progression to ischemic cardiomyopathy at later stages. Altogether, the addition of this innovative biologic system could hopefully photo-power the ischemic myocardium of patients in the future. 
Financial \& competing interests disclosure

The authors have no relevant affiliations or financial involvement with any organization or entity with a financial interest in or financial conflict with the subject matter or materials discussed in the manuscript. This includes employment, consultancies, honoraria, stock ownership or options, expert testimony, grants or patents received or pending, or royalties.

No writing assistance was utilized in the production of this manuscript.

\section{References}

1. Tzahor E, Poss KD. Cardiac regeneration strategies: staying young at heart. Science 356(6342), 1035-1039 (2017).

2. Zwetsloot PP, Vegh AM, Jansen Of Lorkeers SJ et al. Cardiac stem cell treatment in myocardial infarction: a systematic review and meta-analysis of preclinical studies. Circ. Res. 118(8), 1223-1232 (2016).

3. Garbern JC, Lee RT. Cardiac stem cell therapy and the promise of heart regeneration. Cell Stem Cell 12(6), 689-698 (2013).

4. Flaherty MP, Pant S, Patel SV et al. Hemodynamic support with a microaxial percutaneous left ventricular assist device (Impella ${ }^{\circledR}$ ) protects against acute kidney injury in patients undergoing high-risk percutaneous coronary intervention. Circ. Res. 120(4), 692-700 (2017).

5. Cohen J, Goldstone A, Paulsen M et al. An innovative biologic system for photo-powered myocardium in the ischemic heart. Sci. Adv. 3(6), e1603078 (2017).

6. Lehoux S, Levy BI. Collateral artery growth: making the most of what you have. Circ. Res. 99, 567-569 (2006).

7. Dutta P, Sager HB, Stengel KR et al. Myocardial infarction activates CCR2(+) hematopoietic stem and progenitor cells. Cell Stem Cell 16(5), 477-487 (2015).

8. Shiraishi M, Shintani Y, Shintani Y et al. Alternatively activated macrophages determine repair of the infarcted adult murine heart. J. Clin. Invest. 126(6), 2151-2166 (2016).

9. Zhang T, Zhang Y, Cui M et al. CaMKII is a RIP3 substrate mediating ischemia- and oxidative stress-induced myocardial necroptosis. Nat. Med. 22(2), 175-182 (2016). 
